\title{
Investigation of Antibacterial, Antioxidant and UV Resistance Properties of Chitosan, Aloe Vera and Silk Sericin
}

\author{
Joykrisna Saha, Md. Ahsan Habib and Md. Ibrahim H Mondal* \\ Polymer and Textile Research Lab, Department of Applied Chemistry and Chemical Engineering, Rajshahi University, Bangladesh
}

*Corresponding author: Md. Ibrahim H Mondal, Polymer and Textile Research Lab, Department of Applied Chemistry and Chemical Engineering, Rajshahi University, Bangladesh. Email: mihmondal@yahoo.com

Received Date: March 23, 2021

Published Date: May 18, 2021

\begin{abstract}
In this study the physicochemical properties and functional characteristics like antibacterial, antioxidant and UV resistant properties of chitosan, Aloe vera and silk sericin have been investigated. In order to determined different physicochemical and functional properties of chitosan, Aloe vera and silk sericin, antibacterial activity, antioxidant attribute, thermal property, UV protection factor were measured using different standard methods including FTIR, UV-Vis, and XRD instruments. The antibacterial activity was determined against S. aureus gram positive bacteria. Among the materials chitosan solution was shown best antibacterial activity. The antioxidant activity was evaluated by 2,2-diphenyl-1-picrylhydrazyl (DPPH) radicals. The results showed that silk sericin solution had a strong scavenging capacity for DPPH radicals compare to others. The extracted Aloe vera was shown better UV resistant property. The Aloe vera powder was shown highest crystalline diameter by XRD analysis.
\end{abstract}

Keywords: Chitosan; Aloe vera; Silk sericin; Antibacterial activity; Anti-oxidant property; UV protection factor

\section{Introduction}

Chitosan is the second most available biopolymer after cellulose. Chitosan as a result of $\mathrm{N}$-deactylation from chitin and has linear chain of $\beta$ - $(1,4)$ linked 2-acetamino-2-deoxy- $\beta$-D-glucopyranose and 2-amino-2-deoxy- $\beta$-D glucopyranose [1]. Shrimp industries generate large amounts of shrimp bio-waste during processing, approximately $45-55 \%$ of the weight of raw shrimp. Aloe vera (Aloe barbadencis Miller) belong to Liliaceae family. The Aloe vera gel consists of about $99 \%$ water and remaining $1 \%$ solid material consists of a range of compound including vitamins, minerals, polysaccharides, enzymes, phenolic compounds and organic acids. Silk sericin is another natural bio-polymer and water-soluble globular protein that derived from silkworm Bombyx mori. In silk industry huge amount of silk sericin are discarded during degumming process. When this effluent is released from the industry which lead to destroy the environment. It is estimated that 400,000 tons of dry cocoons are produced worldwide per year. After degumming process at least 50,000 tons (12.5\%) of sericin are going into wastewater. This generates a high chemical and biological oxygen demand as well as contamination of water.

Antimicrobials control, destroy or suppress the growth of microorganisms and their negative effects of odor, staining and deterioration. Oxidation is a chemical reaction that can produce free radicals, leading to chain reactions that may damage cells. Consequently, they can cause a wide range of illnesses and chronic diseases. In addition to oxidation is a major problem in food preservation. The antioxidant is a molecule that inhibits the oxidation of other molecules. Ultraviolet radiation up to 60 times as strong as the sun's rays which is harmful for human skin. Generally, UV rays 
absorb by ozone layer. But at present UV rays come to earth easily due to climate changes and depletation of ozone layer which causing skin damages such as sun burn, premature skin ageing, allergies and skin cancer.

In this research three materials like chitosan, silk sericin and Aloe vera are chosen because chitosan and silk sericin are considered as wastage material on the other hand Aloe vera is a very cheap and available material. In addition to these three materials are nontoxic, biologically safe and environment friendly. The aim of this study to investigate the antibacterial, antioxidant, UV resistance and other structural properties of chitosan, Aloe vera and silk sericin.

\section{Experimental}

\section{Materials}

Shrimp shell waste was collected for chitosan production from Khulna sea beach belt. Aloe vera leaves were collected from local market of Rajshahi. Bombyx mori silk cocoons were collected from Bangladesh Sericulture Research Training Institute (BSRTI), Rajshahi. Methanol was used to extract the Aloe vera. Sodium hydroxide, hydrochloric acid and sodium hypochlorite were used for chitosan extraction. Acetic acid was used to dissolve the chitosan All chemicals were purchased from Sigma-Aldrich, BDH, Merck and used without further purification.

\section{Methods}

Chitosan was prepared from shrimp shells accoding to Alam, et al. [2]. Extraction of silk sericin can be accomplished by the method with some modification according Khalifaa, et al. [3]. Aloe vera extraction was done by Ghayempour, et al. [4].

\section{Fourier transform infrared spectrophotometer (FTIR)}

The samples were analyzed with FTIR spectrophotometer (Spectrum-100, Perkin Elmer, USA) in the scanning range of 400$4000 \mathrm{~cm}^{-1}$. The sample was mixed with potassium bromide $(\mathrm{KBr})$ using mortar pestle to make powder in the mass ratio of 1:100 (1 $\mathrm{mg}$ specimen and $100 \mathrm{mg} \mathrm{KBr}$ ). The sample discs were prepared from the powder according to Bagheri-Khoulenjani, et al. [5]

\section{UV Absorption measurement}

The UV absorption spectrum of the sample solution was recorded using UV-visible Spectrophotometer (UV-visible Spectrophotometer, 1650 model, Shimadzu, Japan.

\section{X-Ray diffraction (XRD) analysis}

The XRD patterns were recorded using dry powder samples on PAN Analytical X Pert PRO X-ray diffractometer using $\mathrm{Cu}-\mathrm{K} \alpha$ radiation of wavelength $1.5406 \AA$ as the X-ray source. The measurement was carried out at a scanning rate of $8^{\circ} / \mathrm{min}$ in $2 \theta$ range of $10^{\circ}-60^{\circ}$

The peak width at half maximum (FWHM) in the XRD was used to determine the crystal diameter as per the following Deby-Scherrer formula [6]:

$$
D=\frac{K \lambda}{\beta \operatorname{Cos} \theta}(1)
$$

Where, $\mathrm{K}(=0.9)$ is the Scherrer constant, the X-ray wavelength is $0.15406 \mathrm{~nm}, \beta$ is the peak width of half maximum, and $\theta$ is the Braggs diffraction angle, and $\beta$ is the full width of the X-ray pattern line at half peak-height in radians.

\section{Antibacterial activity test}

Zone of Inhibition of sample solution was determined by disc diffusion method [7]. $250 \mathrm{ml}$ of nutrient agar medium was prepared and autoclaved. The autoclaved nutrient agar medium was poured into sterile petri dish under aseptic conditions. The Petri dish was inoculated with $20 \mu \mathrm{l}$ of $S$. aureus bacteria culture. Discs prepared by punching Whatman paper and autoclaved. Discs were dipped 5 and $10 \mathrm{~g} / \mathrm{l}$ concentration sample solution. The discs were placed on the inoculated petri dish. The petri dish was incubated at $37^{\circ} \mathrm{C}$ for 24 hours and were observed for the formation of zone of inhibition to find the antibacterial activities on the other hand bacterial growth was determined by optical density (OD). The optical density was measured using UV spectrophotometer at $600 \mathrm{~nm}$. The bacterial cell population increases, the transmitted light decreases and absorbance increases.

\section{Anti-oxidant test}

The free radical scavenging activity (RSA) of the samples were evaluated by 2, 2-diphenyl-1-picrylhydrazyl (DPPH) stable free radical according to method of the Shimada, et al. [8]. The free radical scavenging was calculated using the following equation.

$$
(R S A)=\left(1-\frac{\text { Sample absorbance }}{\text { Control absorbance }}\right) \times 100
$$

Control absorbance means methanolic solution of $100 \mu \mathrm{l} D P P H$, and sample absorbance means a mixture of $3 \mathrm{ml}$ methanolic solution of DPPH and $1 \mathrm{ml}$ sample solution.

\section{Measurement of UV protection factor}

In order to measure the UV Protection Factor, a film of the sample was required. To prepare a film, different concentration of sample solution was spread on $10 \mathrm{~mm}$ length and $2 \mathrm{~mm}$ width glass slide. The prepared film was dried at room temperature. The UV Protection Factor (UPF) of film was calculated according to the AATCC test method [9] by the following equation:

$$
U P F=\frac{\sum_{280}^{400} E_{\lambda} \cdot S_{\lambda} \cdot \Delta_{\lambda}}{\sum_{280}^{400} E_{\lambda} \cdot S_{\lambda} \cdot \Delta_{\lambda} \cdot T_{\lambda}}
$$

where, $E_{\lambda}$ is erythermal spectral effectiveness, $S_{\lambda}$, is solar spectral irradiancies, $\lambda$ is the wavelength in $\mathrm{nm}, \mathrm{T}_{\lambda}$ is the spectral transmission of the chitosan film and $\Delta_{\lambda}$ is the measured wavelength intervals in $\mathrm{nm}$. 


\section{Results and Discussion}

\section{FTIR analysis}

Chitosan shows a broad FTIR peak at $3435 \mathrm{~cm}^{-1}$ that indicates $\mathrm{O}-\mathrm{H}$ and $\mathrm{N}-\mathrm{H}$ stretching vibration of chitosan in Figure 1 (a). The characteristic peaks of chitosan observed at $1649 \mathrm{~cm}^{-1}$ and 1592 $\mathrm{cm}^{-1}$ represents the $\mathrm{C}=0$ stretching vibration of acetyl groups and $\mathrm{N}-\mathrm{H}$ bending vibration of amino groups respectively.

The spectra of Aloe vera (Figure 4) was given different polar groups such as $-\mathrm{OH}$ (corresponds to $3265 \mathrm{~cm}^{-1}$ ), $\mathrm{CH}_{2}$ (corresponds to $2922 \mathrm{~cm}^{-1}$ ), CO (corresponds to $1602 \mathrm{~cm}^{-1}$ ), COO-(corresponds to $1401 \mathrm{~cm}^{-1}$ ) in the Figure 1(b). The prominent peak of sericin shows a peak between 1700 and $1600 \mathrm{~cm}^{-1}\left(1602 \mathrm{~cm}^{-1}\right)$ confirming the stretching vibration of the $\mathrm{C}=0$. The Peak at $1580-1510 \mathrm{~cm}^{-1}(1512$ $\mathrm{cm}^{-1}$ ) confirms the presence of N-H bending of amino group in silk sericin structure in the Figure 1(c). Thus, the characteristic peaks confirm the extracted samples are chitosan, Aloe vera and silk sericin (Figure 1)

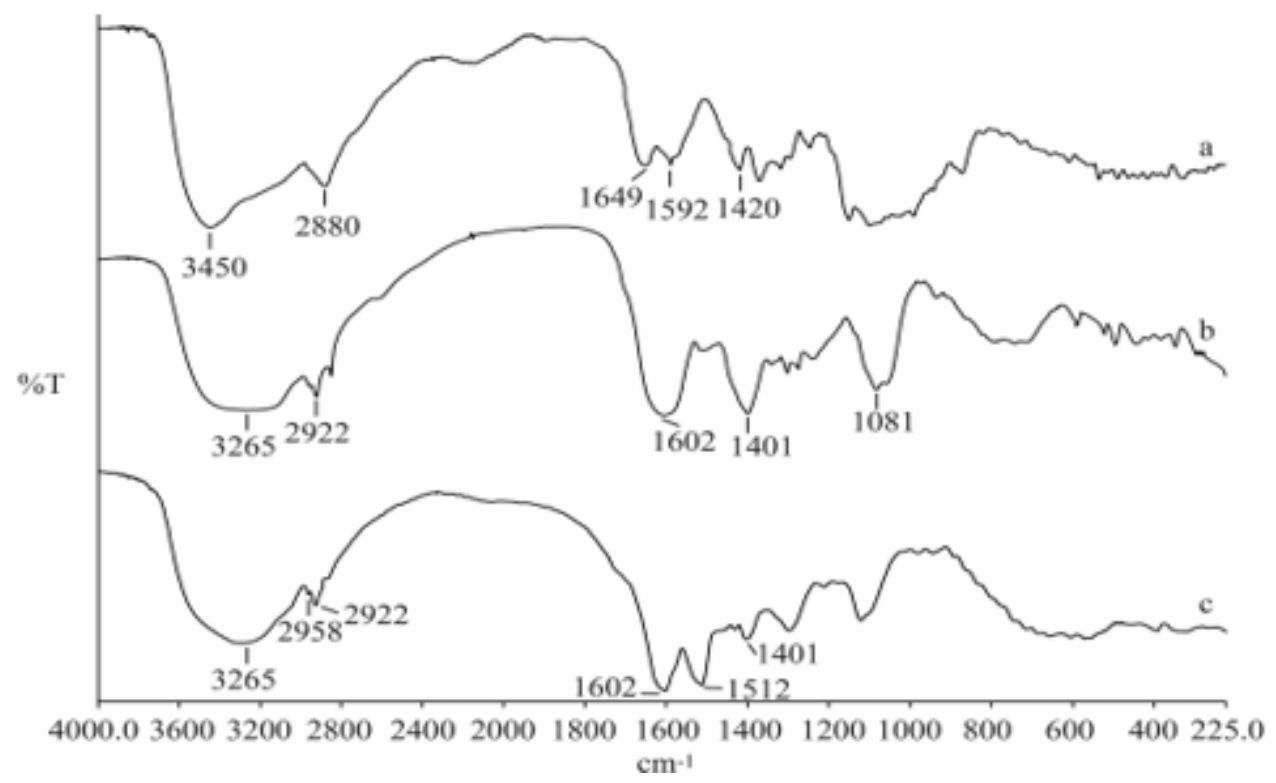

Figure 1: FTIR spectra of (a) Chitosan (b) Aloe vera and (c) Silk sericin.

\section{UV absorption}

Aloe vera extracts showed UV absorption peaks at $359 \mathrm{~nm}$ in the Figure 2 (a). The silk sericin shows a peak absorbance at around
216, 240 and $275 \mathrm{~nm}$ of wavelength in the Figure 2 (b). UV absorption of chitosan peaks was found at $215 \mathrm{~nm}$ in the Figure 2 (c). Silk sericin , Aloe vera and Chitosan were absorbing UV range light. So, all agents may act as UV resistance phenomenon (Figure 2).

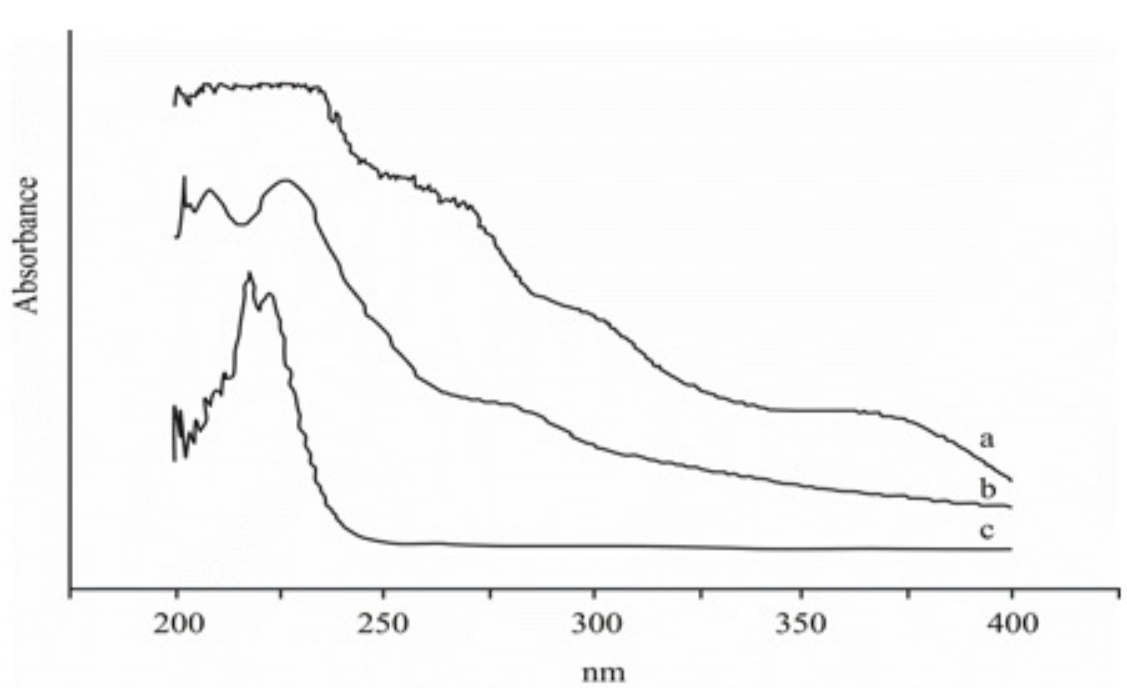

Figure 2: UV Spectra of (a) Aloe vera (b) Silk sericin and (c) Chitosan solution. 


\section{XRD Analysis}

The highest peak intensity was illustrated for Aloe vera sample, shown in the Table 1. On the other hand, the full length at half maximum (FWHM) value of Aloe vera was lowest. As the full length at half maximum (FWHM) value is inversely proportional to the Table 1: Measurement of crystal diameter of the sample.

\begin{tabular}{|c|c|c|c|c|}
\hline Observation & 2 theta value $\left(^{\circ}\right)$ & Peak intensity in count & FWHM ( $\beta$ ) & Crystal Diameter (D) in nm \\
\hline Chitosan & 19.97 & 2773 & 1.369 & 6 \\
\hline Aloe vera & 22.85 & 3564 & 1.276 & 6.4 \\
\hline Silk sericin & 18.86 & 1936 & 1.504 & 5.5 \\
\hline
\end{tabular}

\section{Antimicrobial Activity}

The zone of inhibition of chitosan Aloe vera and silk sericin were evaluated for $10 \mathrm{~g} / \mathrm{l}$ concentration with S. aureus Gram positive bacteria was shown in Figure 3. The zone of inhibition of chitosan was crystal diameter according Deby-Scherrer formula (equation no. 1). When full length at half maximum value decreases crystal diameter of specimen increases. The crystal diameter of Aloe vera was more compare to other specimen because the full length at half maximum value of Aloe vera is minimum (Table 1 ). greater than that of Aloe vera and silk sericin solution. The optical density of bacterial cell population of chitosan was lower than that of Aloe vera and silk sericin solution after 12 hours at same concentration as shown in the Table 2. Lower optical density means higher antimicrobial activity.
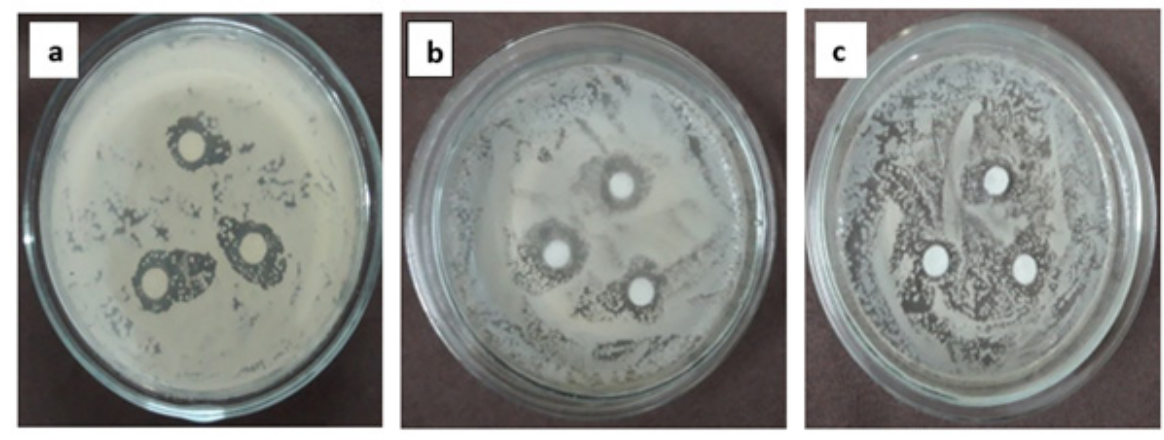

Figure 3: Zone of inhibition of (a) Chitosan (b) Aloe Vera (c) Silk sericin.

The antimicrobial activity of chitosan is due to the interaction of the positively-charged chitosan with the negatively-charged residues on the cell surfaces of bacteria, which causes extensive cell surface alteration and alters cell permeability. This causes the leakage of intracellular substances, such as electrolytes, UV absorbing materials, proteins, amino acids, glucose, and lactate dehydrogenase. As a result, chitosan inhibits the normal metabolism of microorganisms and finally leads to the death of these cells.

\section{Anti-oxidant Activity}

The antioxidant activity increases with the increasing of the concentration of the all samples. The highest scavenging percentage was shown in case of $10 \mathrm{mg} / \mathrm{ml}$ sericin solution compare to other solution as shown in the Table 3. Silk sericin acts as antioxidative agent as the residual free $-\mathrm{NH}_{2}$ groups of sericin can react with that the free radicals (DPPH solution) to form stable molecules and the $-\mathrm{NH}_{2}$ groups can form ammonium groups $\left(\mathrm{NH}_{4}^{+}\right)$by capturing a hydronium ion from the solution.

\section{UV Protection Factor}

From the Table 3, it can be seen that that the UV protection factor of Aloe vera extraction film was the best compare to other material. UV protection factor increases with the increase of concentration for the all samples. Chitosan contains two chromophoric groups, $\mathrm{N}$-acetylglucosamine and glucosamine. These are obtained through partial deacetylation of chitin. These chromophoric groups assist in UV resistance.

Table 2: Optical density of different concentration of solution after 12 hours.

\begin{tabular}{|c|c|}
\hline Observation & OD \\
\hline Control & 1.298 \\
\hline $0.5 \%$ chitosan solution & 0.803 \\
\hline $1 \%$ chitosan solution & 0.721 \\
\hline
\end{tabular}




\begin{tabular}{|c|c|}
\hline $0.5 \%$ Aloe vera solution & 0.891 \\
\hline $1 \%$ Aloe vera solution & 0.844 \\
\hline $0.5 \%$ sericin solution & 0.996 \\
\hline $1 \%$ sericin s solution & 0.942 \\
\hline
\end{tabular}

Table 3: Antioxidant and UV protection value.

\begin{tabular}{|c|c|c|}
\hline Observation & RSA (\%) & UPF rating \\
\hline $5 \mathrm{mg} / \mathrm{ml}$ Chitosan & 25 & 3.64 \\
\hline $10 \mathrm{mg} / \mathrm{ml}$ Chitosan & 32 & 4.46 \\
\hline $5 \mathrm{mg} / \mathrm{ml}$ Aloe vera & 38 & 8.23 \\
\hline $10 \mathrm{mg} / \mathrm{ml}$ Aloe vera & 46 & 10.4 \\
\hline $5 \mathrm{mg} / \mathrm{ml}$ sericin & 56 & 6.3 \\
\hline $10 \mathrm{mg} / \mathrm{ml}$ sericin & 62 & 7.4 \\
\hline
\end{tabular}

\section{Conclusion}

Chitosan, Aloe vera and silk sericin have been illustrated anti-oxidant property, UV resistant and antibacterial properties. As these materials absorb UV range light, they may be used in sunscreen cream and can also be applied on textiles to produce UV protective garments. Chitosan, Aloe vera and silk sericin have been shown antibacterial activity which will be used to produce medical textiles such as surgical suture, hand gloves, gauge, bandage apron and bed sheet. These materials have been also shown an antioxidant property. This valuable ingredient might be used as a food preservative, and in cosmetic and pharmaceuticals sector. It is hope that these wastage and cheap material would helpful for our economy and environment. It is hope that these wastage and cheap materials can be used as value added products for the above-mentioned applications and would be helpful to our national economy. At the same time environment pollution from this waste materials can also be minimized.

\section{Acknowledgement}

None.

\section{Funding}

The authors would like to acknowledge the Faculty of Engineering, Rajshahi University, Bangladesh for funding the Project Ref. No.: 1208/5/52/RU/Eng-10/2019-2020.

\section{Conflicts of interest}

The authors declare that there are no conflicts of interest.
1. dos Santos ZM, Caroni ALPE, Pereira MR, da Silva DR, Fonseca JLC (2009) Determination of deacetylation degree of chitosan: a comparion between conductometric titration and CHN analysis. Carbohydrate Research 344(18): 2591-2595

2. Khalifaa IB, Ladharib N, Touayc M (2012) Application of sericin to modify textile supports. The Journal of the Textile Institute 103(4): 370-377.

3. Alam , Khan MA, Khan RA, Ghoshal S, Mondal MIH (2008) Study on the physico mechanical properties of photo-cured chitosan films with oligomer and acrylate monomer. Journal of Polymer and Environment 16: 213-219.

4. Ghayempour S, Montazer M, Rad MM (2016) Simultaneous encapsulation and stabilization of Aloe vera extract on cotton fabric for wound dressing application. RSC Advances 6: 111895-111902.

5. Bagheri-Khoulenjani S, Taghizadeh H, Mirzadeh (2009) An investigation on the short-term biodegradability of chitosan with various molecular weights and degrees of deacetylation. Carbohydrate Polymers 78(4): 773-778.

6. Wang Y, Chang Y, Yu L, Zhang C, Xu X, et al. (2013) Crystalline structure and thermal property characterization of chitin from Antarctic krill (Euphausia superba). Carbohydr Polym 92(1): 90-97.

7. AATCC Test Method: TM 147-2004 (2010) Determination of Zone of Inhibition by Qualitative Method. AATCC Technical Manual, pp. 251-252.

8. Shimada K, Fujikawa K, Yasara K, Nakamura T (1992) Antioxidative properties of xanthan on the oxidation of soya bean oil in cyclodextrin emulsion. Journal of Agriculture and Food Chemistry 40: 945-948.

9. AATCC Test Method: TM 183-2004 (2021) Transmittance or blocking of erythemal weighted ultraviolet radiation through fabric. AATCC Technical Manual, AATCC Technical Manual, Research Triangle Park, NC, USA 85: 318-321.

\section{References}

